\title{
The New Algorithm of Fingerprint Reference Point Location Based on Identification Masks
}

\author{
Piotr Porwik ${ }^{1}$ and Krzysztof Wrobel ${ }^{2}$
}

1 Institute of Informatics, Silesian University, 41-200 Sosnowiec, ul. Bedzinska 39 porwik@us.edu.pl

2 Institute of Informatics, Silesian University, 41-200 Sosnowiec, ul. Bedzinska 39 kwrobel@zsk.tech.us.edu.pl

\section{Introduction}

Fingerprint identification is one of the most important biometric technologies especially in Fingerprint Identification System (AFIS). A fingerprint is the pattern of ridges and valleys on the surface on a fingertip. Each human has unique fingerprints. The uniqueness of a fingerprint allows to build systems which can recognize personal fingerprint feature automatically. Unfortunately it is a difficult task, because fingerprint images have very poor quality very often. In practice, due to variations in impression conditions, ridges configuration, skin condition, acquisition devices, etc. many restrictions have to considered. The ridge structures in poor-quality fingerprint images are not always well-defined, therefore they cannot be detected. Fortunately a fingerprint experts are often able to correct such corrupted images as long as the ridges and valleys structure is not corrupted completely. Each analyzed digital fingerprint image should has area where structure of finger will be visible. Generally, fingerprint images can be divided into three types: well-defined region, recoverable corrupted region and unrecoverable region $[1,2,7]$. In the first region ridges and valley are clearly differentiated, in the second region ridges and valleys are corrupted by a small amount of creases or smudges but are still visible. In the third area ridges and valleys are strong corrupted - such image is rejected. In our paper only two first areas are analyzed and each of them can be used to fingerprint recognition. In practice, fingerprint recognition and classification based on two techniques: the reference point location and the Gabor filtering or the ridge characteristics (called minutiaes). In this paper a new method of the reference point alignment has been presented. A new approach of reference point localization is based on so-called identification masks which have been composed on the basis of analysis of biometric characteristic of human finger. Construction of such masks has been presented. 


\section{Fingerprint enhancement}

Fingerprint enhancement is used to recover the topology structure of ridges and valleys from the noisy image. An image enhancement allows to improve quality of digital fingerprint representation but this method should not results in any spurious ridge structures and configurations. This assumption is important because any artificial ridges deformation may change the base imprint. In fingerprint processing two types of images are accepted: binary or gray-level images. The binary image can be obtained of course from gray-level image. Unfortunately in binarization procedures some information about ridges can be lost therefore a gray-level images are preferred. The more advanced fingerprint enhancement procedure is described in [1]. In mentioned paper among other things orientation image, ridge frequency image and filtering have been explained. Such description for fingerprint minutiae's has been dedicated but can also be used for our experiments. A gray-level image $I$ is defined as $N \times N$ matrix where $P(x, y)$ represents the intensity of pixel at the $(x, y)$ point. In presented implementation, fingerprint image has dimension $256 \times 256$ and with 500 dpi resolution have been scanned. Such resolution is recommended by FBI. In the first stage a gray-level image to black-white format is exchanged, where the binarization threshold has been fixed on the level:

$$
T=\frac{1}{N^{2}} \sum_{x=0}^{N-1} \sum_{y=0}^{N-1} P(x, y)
$$

The constant 30 which occurs in equation (1) was fixed experimentally, and allows us to get the better binarization effect for scanned fingerprint images with dark background.

\section{Direction image determination}

In general, analyzed fingerprint is a well-defined orientation field, which has to compared with pattern. In most cases the reference point detection methods operate on the orientation field of fingerprint. The Poincare index analysis is well known method described in [5]. Unfortunately, this method is sensitive to noise of orientation field. Very efficient method proposed in [2] is based on multiresolution analysis, but this method is sensitive to the fingerprint rotation. In presented method, the orientation field, with some additional modifications, to reference point indication has been exploited. In our approach, base on orientation field, the special masks are proposed, which allow to detect a unique reference point consistently and accurately for all types of prints. To decide the ridge direction of each pixel in the image, the ridge direction of a given pixel $P(x, y)$ into directions $i(i=0,1, \ldots, 7)$ has been divided in a $9 \times 9$ window with the pixel in the center [3] (Fig. 1b). The gray values of pixels in eight directions (at positions marked by numbers $0,1, \ldots, 7$ ) are added 
together to obtain sums $s_{0}, s_{1}, \ldots, s_{7}$. The sums $s_{i}$ are equivalent to convolving the image with $9 \times 9$ masks, where each mask has value of 1 at positions where block shown in Fig. 1b has values $i$ and 0 elsewhere. In next stage, for indices $i \mathrm{~min} / \mathrm{max}$ values are estimated:

$$
i=\left\{\begin{array}{clll}
s_{d}=\underset{i=\{0,1, \ldots, 7\}}{\arg }\left\{\max \left(s_{i}\right)\right\} & \text { for } & p_{x y}^{\text {bin }}=1 \\
s_{l}=\underset{i=\{0,1, \ldots, 7\}}{\arg }\left\{\min \left(s_{i}\right)\right\} & \text { for } & p_{x y}^{b i n}=0
\end{array}\right.
$$

where: $p_{x y}^{b i n}$ is a pixel value at point $(x, y)$, in fingerprint digital image.

The direction at pixel is defined by means of $s_{d}$ value if the central pixel is located on a ridge (black area), and by the $s_{l}$ value if the central pixel is located in a valley. From equation (2), indices image can be prepared. Such image can also be called direction image. Unfortunately, obtained values treated as direction for each pixel are usually noisy, therefore they should be smoothed and averaged in a local neighborhood. In our application, as smoothing operation the mode function has been applied. The function mode computes the mode of the given data and the mode is defined as observation with the highest frequency. In other words, mode function can be treated as measure of central tendency. For example $d=\operatorname{mode}(a, b, c, d, d, d, e, f, g)$. If there is more than one observation with the modal frequency, then choice is arbitrary. Results of mentioned operations are shown on Fig. 1.

a)

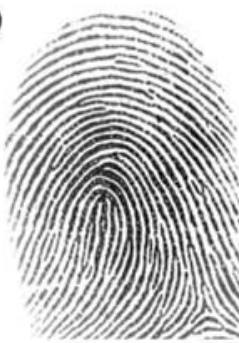

b)

b)

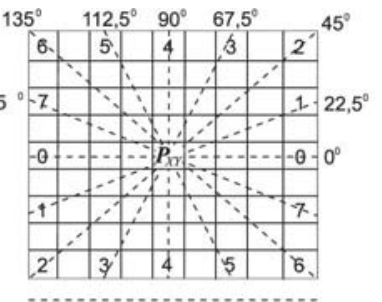

c) d)

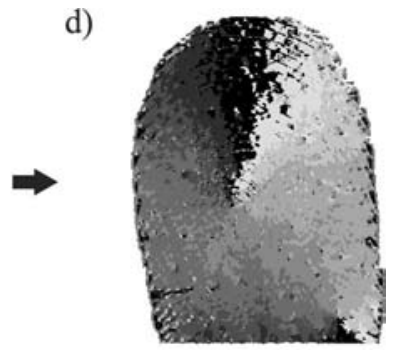

$\mathrm{d}=\operatorname{mode}(\mathrm{a}, \mathrm{a}, \mathrm{d}, \mathrm{d}, \mathrm{d}, \mathrm{d}, \mathrm{b}, \mathrm{b}, \mathrm{c})$

Fig. 1 .

a) a given fingerprint, b) the $9 \times 9$ mask to compute the ridge directions, c) $3 \times 3$ mode filter mask, d) directions image.

\section{The reference point determination by means of masks}

For the first time, described bellow method in [7] has been presented. In present paper that method has been extended and new researches were car- 
ried out. From directions image, the orientation field has been prepared $[2,4]$. The orientation represents the local orientation of the ridges contained in the fingerprint. Directions image from Fig. 1d into $9 \times 9$ blocks (windows) has been divided. In each block, dominant direction (the most frequently occurring direction) like previously was calculated. From the gradient information the orientation angle is estimated. The 8 directions (see Fig. 1b) in each block is stored as table. This table can be presented as image (although it is not necessary), where each direction is performed as appropriate line. For example, for angle $0^{\circ}$ horizontal line is performed, for angle $90^{\circ}$ - vertical, and so one by step $22.5^{\circ}$ for another direction lines. In next stage background is eliminated and only foreground is used. If in a given block $9 \times 9$ at least one black pixel can be found, then whole block create part of orientation field, otherwise such block is treated as background and is rejected. After background elimination

a)

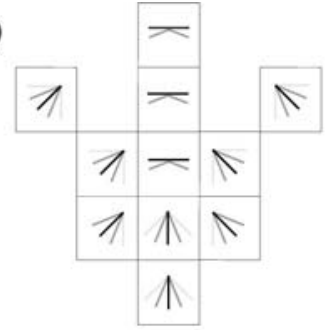

c)

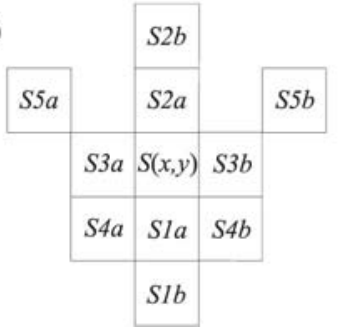

b)

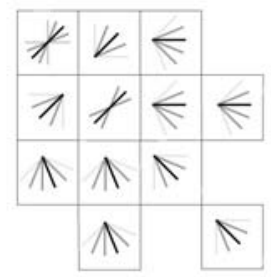

d)

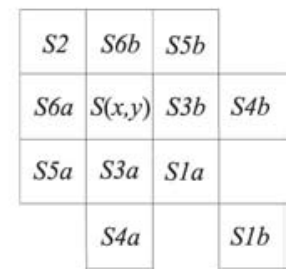

Fig. 2.

a,b) features of identification mask,

c,d) the identification masks for $0^{\circ}$ and $+45^{\circ}$, respectively

the orientation field is smoothing with the aid of mode mask, similar as previously. In the last stage the reference point is determined. Because print of finger can be impressed in different manner, influence of rotation should be eliminated. In our method orientation field image is filtered by means of appropriates masks. That masks have special construction, what allows to collect fingerprints located in $0^{0}, \pm 45^{0}, \pm 90^{\circ}$. Proposed masks are called identification masks. Fig.2a,b present identification features of each mask. A special arrangement of short lines in each cell characterizes ridges in a human fingerprint. Such ridges distribution has been established experimentally. Such 
approach is very efficient and it is equivalent to minutiae detection method $[5]$, but unlike another methods $[1,4,6]$ our solution gives faster reference point detection. By means of identification masks, which explore whole orientation field, fingerprint features represent by appropriate mask are sought. The masks for angles $0^{\circ}$ and $45^{\circ}$ present Fig. 2c,d. Because it is not known, how fingerprint was impressed, orientation field is filtered in turn by all masks. Finally, each identification mask more than one reference point can indicate. All identification masks into cells are split. The cell indicate direction at point $x, y$ in orientation field. The 8 directions in orientation field are represented by means of numbers $0,1,2,3,4,5,6,7$. Such numbers are substitution of angles $0^{0}, 22.5^{0}, 45^{0}, 67.5^{0}, 90^{0}, 112.5^{0}, 135^{0}, 157.5^{\circ}$, respectively (see Fig. 1b).

From orientation field (Fig. 2) the reference point for the angle $0^{\circ}$ can be determined if the global condition is fulfilled:

$$
\begin{aligned}
& \text { if }((S(x, y)=0) \text { or }(S(x, y)=1) \operatorname{or}(S(x, y)=7)) \text { and } \\
& (((S 1 a=2) \operatorname{or}(S 1 a=3) \operatorname{or}(S 1 a=4) \operatorname{or}(S 1 a=5) \text { or }(S 1 a=6)) \text { and } \\
& ((S 1 b=2) \operatorname{or}(S 1 b=3) \operatorname{or}(S 1 b=4) \operatorname{or}(S 1 b=5) \operatorname{or}(S 1 b=6))) \text { and } \\
& (((S 2 a=0) \operatorname{or}(S 2 a=1) \operatorname{or}(S 2 a=7)) \text { and } \\
& ((S 2 b=0) \operatorname{or}(S 2 b=1) \operatorname{or}(S 2 b=7))) \text { and } \\
& (((S 3 a=0) \operatorname{or}(S 3 a=1) \operatorname{or}(S 3 a=2) \operatorname{or}(S 3 a=3) \operatorname{or}(S 3 a=4)) \text { and } \\
& ((S 3 b=0) \operatorname{or}(S 3 b=4) \operatorname{or}(S 3 b=5) \operatorname{or}(S 3 b=6) \operatorname{or}(S 3 b=7))) \text { and } \\
& (((S 4 a=1) \operatorname{or}(S 4 a=2) \operatorname{or}(S 4 a=3) \operatorname{or}(S 4 a=4)) \text { and } \\
& ((S 4 b=4) \operatorname{or}(S 4 b=5) \operatorname{or}(S 4 b=6) \operatorname{or}(S 4 b=7)) \text { and } \\
& (((S 5 a=0) \operatorname{or}(S 5 a=1) \operatorname{or}(S 5 a=2) \operatorname{or}(S 5 a=3) \text { or }(S 5 a=4)) \text { and } \\
& ((S 5 b=0) \operatorname{or}(S 5 b=4) \operatorname{or}(S 5 b=5) \operatorname{or}(S 5 b=6) \operatorname{or}(S 5 b=7)))
\end{aligned}
$$

For remained masks, performed condition (3) should be modified by appropriate rotation of the masks. In the worst case, each identification mask can point different reference points, but it is well known that for fingerprint only one reference point can be indicated. From this reason, for all potential reference points, detected by means of identification masks (Fig. 2c), so-called influence coefficients have been estimated. The influence coefficients (inco) is calculated as follow:

For angle $0^{0}$ :

$$
\begin{aligned}
& \text { if }(S(x, y)=S 2 a) \text { then inco }:=\text { inco }+1 \text {; } \\
& \text { if }(S 5 a<>4) \text { or }(S 5 b<>4) \text { then inco }:=\text { inco }+1 \text {; } \\
& \text { if }(|S 1 a-S(x, y)|=4) \text { or }(|S 1 b-S(x, y)|=4) \text { then inco }:=\text { inco }+1 \text {; }
\end{aligned}
$$

For angle $+45^{\circ}$ :

$$
\begin{aligned}
& \text { if }(S(x, y)=S 2) \text { then inco }:=\text { inco }+1 \\
& \text { if }(S 5 a \leq 1) \text { or }((S 5 b \geq 4) \text { and }(S 5 b \leq 6)) \text { then inco }:=\text { inco }+1 \\
& \text { if }(|S 1 a-S(x, y)|=4) \text { or }(|S 1 b-S(x, y)|=4) \text { then inco }:=\text { inco }+1 \text {; }
\end{aligned}
$$

Remained the influence coefficients are calculated similarly, and only appropriate values of angles should be changed: for $-45^{\circ}, \pm 90^{\circ}$, respectively. Additionally, the next principles are considered: 
- if two (or more) references points is located in a local neighborhood, then value of the inco coefficient is increased of 4 ,

- for the lowest located reference point, its inco value is increase of 5,

- potential reference point which lies at a distance less than 8 pixels from edge background is rejected.

Finally, the point which has the largest inco coefficient, will be classified as reference point. Proposed detection of reference point together with the $2 D$ Gabor filtering method $[1,2]$ can be used to fingerprint matching.

\section{Experimental results}

In experiments fingerprint images from FVS database have been used [9]. Two type experiments has been conducted. The first experiment by means of graphic form has been performed. Fig. 3 presents results of such experiment, where various fingerprint images have been compared. Such images with two methods have been tested. The first method is described in this paper. The second method is full described in [8], where MatLab program is included. Such program based on method presented in [2]. Fig. 3 presents differences between reference point location. The reference points by signs ' $t^{\prime}$ or ' $x$ ' have been marked. Our reference point location by means of ' $x$ ' sign is indicated. The results of method $[2,8]$ as ${ }^{\prime}{ }^{\prime}$ is marked. The results of the second experiment presents Table 1 . In this table reference point is defined by point $(x, y)$, where $x, y$ are pixels coordinates. Mentioned point by three manner has been determined. The column $A$ by police expert has been stated. All values i Table 1 should be interpreted as pairs of numbers, which indicate appriopriate $(x, y)$ coordinates. The values in the columns $B$ and $C$ by algorithm $[2,8]$ and our method have been computed. The columns $D 1, D 2$, and the last column show differences between methods. Presented differences it is the Euclidean distance between appropriate $x$ and $y$ coordinates. For example distance in the column $D 1$ is computed from formula $\left[\left(x_{a}-x_{b}\right)^{2}+\left(y_{a}-y_{b}\right)^{2}\right]^{0.5}$. The values in the column $D 2$ are calculated similarly. The last column shows difference between methods. From obtained results follow (all positive values) that in most of the cases our method gives the better results. In other words reference point location lies closer to expert's reference point.

\section{Conlusions}

In this paper, a new method to locate a unique reference point has been proposed. Since human experts may not be able to locate the pixel wise accurate reference point, we propose the new method which allow to determine such point. The our method base on so-called identification masks, which was designed on the basis of human finger print analysis. For proposed masks, the 
Table 1. Results of fingerprint identification

\begin{tabular}{|c|c|c|c|c|c|c|}
\hline Image & $\begin{array}{l}\text { Expert's } \\
\text { assesment } \\
\left(x_{a}, y_{a}\right) \\
\text { A }\end{array}$ & $\begin{array}{c}\text { Alghoritm } \\
{[8]} \\
\left(x_{b}, y_{b}\right) \\
\mathrm{B}\end{array}$ & $\begin{array}{c}\text { Our } \\
\text { method } \\
\left(x_{c}, y_{c}\right) \\
\mathrm{C}\end{array}$ & $\begin{array}{l}\text { Distance } \\
D 1= \\
A-B\end{array}$ & $\begin{array}{l}\text { Distance } \\
D 2= \\
A-C\end{array}$ & $D 1-D 2$ \\
\hline $19 \_7 . \mathrm{bmp}$ & 125,111 & 120,136 & 130,94 & 25,50 & 17,72 & 7,78 \\
\hline $37^{-} 3 . \mathrm{bmp}$ & 153,92 & $146, \pm 06$ & 157,85 & 15,65 & 8,06 & 7,59 \\
\hline $37^{-} 5 \_2 . \mathrm{bmp}$ & 143,144 & 152,167 & 139,139 & 24,70 & 6,40 & 18,30 \\
\hline $37_{-}^{-} 7 \bar{b} \mathrm{mp}$ & 116,102 & 96,151 & 121,94 & 52,92 & 9,43 & 43,49 \\
\hline $1-1 . b m p$ & 168,160 & Error & 167,149 & - & 11,05 & - \\
\hline $1 \overline{1} \_1 . \mathrm{bmp}$ & 115,153 & 123,153 & 112,148 & 8,00 & 5,83 & 2,17 \\
\hline
\end{tabular}

influence coefficients have been stated. Proposed method with complete algorithm described in $[2,8]$ has been compared. The described method with the aid of the $F V S$ fingerprint database has been tested. Mentioned data collection includes varying quality fingerprint images.

a)

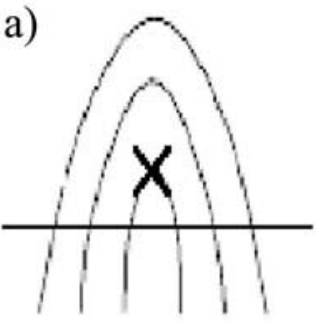

d)

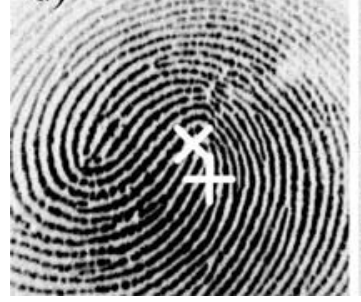

b)

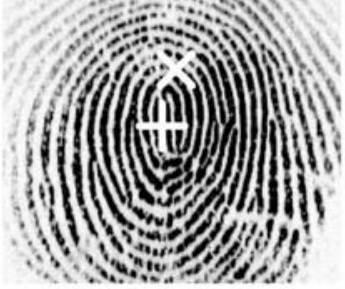

e)

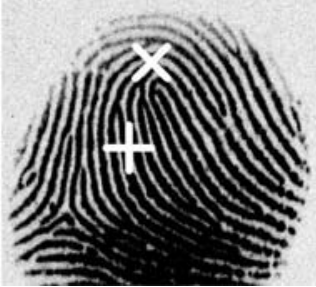

c)
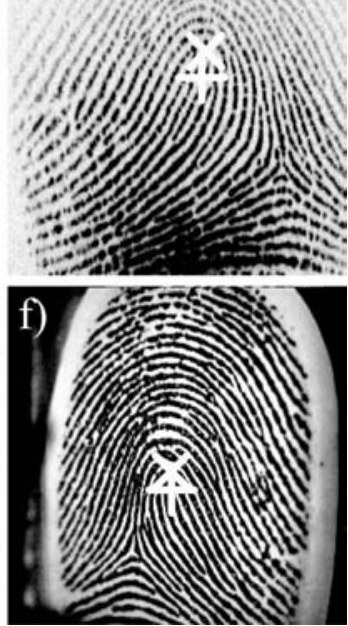

Fig. 3.

a) The perfect reference point location,

b)-f) reference point determination for different fingerprints quality from the $F V S$ database. 


\section{References}

1. L. Hong, Y. Wan, A.K. Jain (1998) Fingerprint image Enhancement: Algorithm and Performance Evaluation. IEEE Trans. On Pattern Analysis and Machine Intelligence, vol. 20, no.8, pp. 777-789.

2. A.K. Jain, S. Prabhakar, L. Hong, S. Pankanti (2000) Filterbank-Based Fingerprint Matching. IEEE Trans. on Image Processing, vol. 9, no. 5.

3. A. K. Jain, S. Prabhakar, L. Hong (1999) A multichannel approach to fingerprint classification. IEEE Trans. Pattern Analysis and Machine Intelligence, Vol. 9 , no. 4 pp. 348-359.

4. X. Jiang, M. Liu, A.C., Kot (2004) Reference Point Detection for Fingerprint Recognition. Proc. of the 17th Int. Conf. On Pattern Recognition (ICPR'04) Cambridge, pp. 540-543, UK.

5. K. Karu, K. Jain (1996) Fingerprint Classification. Pattern recognition, vol.29, no. 3, pp. 389-404.

6. C.H. Park, S.K. Oh, D.M. Kwak, B.S. Kim, Y.C., Song, K.H. Park (2003) A New Reference Point Detection Algorithm Based on Orientation Pattern Labeling in Fingerprint Images. Proc. of 1st Iberian Conf. On Pattern Recognition and Image Analysis, Puerto de Andratx, pp.697-703, Spain.

7. P. Porwik, L. Wieclaw (2004) A new approach to reference point location in fingerprint recognition. IEICE International Journal Electronics Express Vol. 1, No. 18, pp. 575-581, Japan.

8. Luigi Rosa (2004) Fingerprint Recognition System, http://www.mathworks.it/matlabcentral/fileexchange/loadFile.do ?f4addg_fingerprint\&objectId $=4239 \&$ objectType $=$ file

9. Shivang Patel (2002-2004) Fingerprint Verification System, http://fvs.sourceforge.net/download.html 